\title{
Prevalence of variation in predicted refraction between different intraocular lens formulae
}

\author{
Keith Ong ${ }^{1,2}$, Linda Feng ${ }^{3}$ \\ ${ }^{1}$ Royal North Shore Hospital Department of Ophthalmology, University of \\ Sydney, Sydney, Australia; '2Dalcross Adventist Hospital, Sydney, Australia; \\ ${ }^{3}$ Nepean Hospital, Sydney, Australia
}

\begin{abstract}
Variations of $0.5 \mathrm{D}$ in predicted refraction between the different intraocular lens (IOL) calculation formulae may occur in $19.4 \%$ cases. This has implications when reporting refractive surprise. It also shows that it is beneficial to consider more than one $1 O L$ formula when choosing IOL power for cataract surgery.
\end{abstract}

Selecting the intraocular lens $(\mathrm{IOL})$ power for cataract surgery can be a dilemma when there is a variation in predicted refraction between the different IOL formulae for a particular power of IOL.

The amount of refractive surprise (RS) can vary depending on which IOL calculation formula is used when there is a variation in predicted refraction. A small study was done to evaluate the prevalence and extent of this variation.

Four intraocular lens calculation formulae were compared: SRK/T, Haigis, Holladay 1, and Holladay 2. Zeiss IOL Master 500 was used. The cohort consisted of 105 eyes of 64 patients who had cataract phacoemulsification and insertion of posterior chamber IOL (Alcon SN60WF) in the months of January to June 2015.

The incidence and amount of discrepancy in predicted refraction between the different IOL formulae and their respective refractive surprise were analysed. The degree of variation was stratified into three groups: $<0.25 \mathrm{D} ; 0.25$ to $0.5 \mathrm{D}$; and $>$ $0.5 \mathrm{D}$. A variation of $<0.25 \mathrm{D}$ was found in $46.2 \%$ cases. In $34.4 \%$ of cases, the variation was between 0.25 to $0.5 \mathrm{D}$. A discrepancy of $>0.5 \mathrm{D}$ in $\mathrm{IOL}$ formula calculation was found in $19.4 \%$ of cases.

Hence, $19.4 \%$ of cases in this cohort had a variation of $>0.5 \mathrm{D}$ in predicted refraction between the different IOL formulae. These cases were found to have axial length, anterior chamber depth, or keratometer readings that were beyond average, as well as anterior chamber depth disproportionate to axial length.

Variations in predicted refraction between different IOL formulae would be significant if it were $>0.5 \mathrm{D}$, because this could contribute to refractive surprise. Refractive surprise in this study was the difference between actual refraction

Correspondence: Dr. Keith Ong, 2 Railway Avenue, Eastwood NSW 2122, Australia.

E-mail: keithong@optusnet.com.au 
two months after cataract surgery compared to the predicted refraction by the IOL formulae for that particular IOL used. Analysing the postoperative refractive results in the $>0.5 \mathrm{D}$ group, we found the $\mathrm{IOL}$ formula that gave the least refractive surprise was Haigis in $50 \%$ of cases, Holladay 2 in $25 \%$ of cases, Holladay 1 in $15 \%$ of cases, and SRK/T in $10 \%$ of cases. This indicates that it is beneficial to consider more than one IOL formula when choosing $\mathrm{IOL}$ power in these cases as it appears that a particular formula may be more suitable for a particular eye. Therefore, when reporting refractive surprise, one has to consider the IOL calculation formula that was used for a particular patient.

A way to minimise the impact of variation in refractive prediction on refractive surprise is to find the formula that results in the least refractive surprise in the first cataract operation and then use this formula for $\mathrm{IOL}$ calculation when the second eye is being operated. 